\title{
Matériaux d'écrivains : conversation avec Raharimanana et Sami Tchak
}

Discutants : Julie Peghini et Nicolas Martin-Granel

\section{Raharimanana et Sami Tchak}

\section{(2) OpenEdition}

\section{Journals}

Édition électronique

URL : https://journals.openedition.org/coma/4042

DOI : $10.4000 /$ coma.4042

ISSN : 2275-1742

Éditeur

Institut des textes \& manuscrits modernes (ITEM)

Référence électronique

Raharimanana et Sami Tchak, "Matériaux d'écrivains : conversation avec Raharimanana et Sami Tchak », Continents manuscrits [En ligne], 12 | 2019, mis en ligne le 16 août 2019, consulté le 14 janvier 2023. URL : http://journals.openedition.org/coma/4042 ; DOI : https://doi.org/10.4000/coma.4042

Ce document a été généré automatiquement le 14 janvier 2023.

\section{(c) (†) $\odot$}

Creative Commons - Attribution - Pas d'Utilisation Commerciale - Pas de Modification 4.0 International - CC BY-NC-ND 4.0

https://creativecommons.org/licenses/by-nc-nd/4.0/ 


\title{
Matériaux d'écrivains : conversation avec Raharimanana et Sami Tchak
}

\author{
Discutants : Julie Peghini et Nicolas Martin-Granel
}

\author{
Raharimanana et Sami Tchak
}

1 La rencontre s'est tenue le 15 février 2019, à l'École normale supérieure de la rue d'Ulm, autour du dernier livre autobiographique des deux écrivains, centré sur la figure du père ; respectivement Revenir (Rivages, 2018) et Ainsi parlait mon père (JC Lattès, 2018).

\section{Raharimanana}

\begin{abstract}
JULIE PEgHiNı - Tu parles souvent, Jean-Luc, de ta culture antakarana ou de la tradition orale du kabary et du hainteny propre à Madagascar, et l'on sent à quel point cette culture et cette tradition sont présentes dans tes textes.

Raharimanana - Cette culture, ces formes traditionnelles que tu évoques se retrouvent en effet dans mon théâtre. J'ai toujours voulu faire du théâtre, même si les textes des auteurs francophones sont peu présents dans le répertoire dramatique français. Il y a comme une sorte de mur à escalader pour qu'un auteur francophone soit accepté tel quel, qu'il ne soit pas un conteur, ou quelque chose de ce genre, sorti de l'oralité ou de l'engagement politique. Quant à être metteur en scène, n'y songez pas! Dans ce contexte, il me fut compliqué de trouver ma place dans le théâtre français, d'autant plus compliqué que je ne voulais pas me contenter d'être dans le théâtre francophone ; cela n'a vraiment pas de sens pour moi. D'un point de vue politique, que signifie faire du théâtre francophone ou se dire francophone? Je ne voulais pas que mon travail artistique soit ramené au seul débat sur la francophonie. J'estime être au cœur d'une fabrique artistique, et non au cœur d'une fabrique d'idéologie ou d'un positionnement politique. Ma rencontre avec le metteur en scène Thierry Bedard a permis que ce soit quelqu'un d'autre qui porte mes idées. Par la suite, j'ai eu plus de facilité à entrer dans le théâtre français. Toutefois, je ne découvre pas aujourd'hui cette tendance à éclater les genres, elle a toujours été là.
\end{abstract}


JULIE PEghiNı - As-tu fondé une compagnie de théâtre?

Raharimanana - En 2014, j'ai créé la compagnie SoaZara, basée à Tours, nous avons créé Rano, rano (Théâtre des bambous à La Réunion), Parfois le vide (Théâtre Antoine Vitez à Ivry, RFI, Festival d'Avignon), et d'autres formes plus légères, des contes et des lectures musicales (Les Contes de la grande île, Par la nuit, etc.). Avant la création de cette compagnie, Thierry Bedard a créé quatre de mes pièces, et l'une d'elles, 47, a été déterminante dans ma réflexion pour créer ma propre compagnie. Rano, rano, est le prolongement du travail mené avec Thierry Bedard autour de l'insurrection de 1947 et de la collecte de témoignages des anciens rebelles. Mais pour cela, il me fallait assumer le projet du début à la fin, c'est pour ça que j'ai créé la compagnie SoaZara.

NICOLAS MARTIN-GRANEL - Nous sentons que la question de la francophonie est un peu douloureuse pour toi. À ce propos, pourrais-tu revenir sur ton départ de Madagascar provoqué justement par l'une de tes pièces qui a bien failli être à l'origine d'une crise diplomatique avec la France?

Raharimanana - Cette question de la francophonie ne m'est pas douloureuse, elle est juste chiante (je m'excuse du terme mais c'est le sentiment qui m'habite le plus lorsqu'on parle de francophonie, il y a comme une sorte d'obsession des critiques sur cette question, et j'ai l'impression de fournir des explications à vie, comme si on ne peut pas passer à autre chose, mais ce n'est pas grave, expliquons à nouveau). En 1988, tout jeune alors, j'ai écrit la pièce Le Prophète et le Président. Nous répétions dans les locaux de l'Alliance française. Dans ces années-là, le régime politique malgache était dictatorial. Deux ans plus tard, François Mitterrand prononçait son célèbre discours à La Baule demandant aux chefs d'État africains d'entrer dans l'ère démocratique. La fin des années 1980 était donc une période charnière et j'ai écrit cette pièce pour dire que, désormais, à Madagascar, on était en démocratie, ce qui, bien sûr, nous a valu quelques soucis de la part des ministères ! Des gens arrivaient en pleine répétition pour acheter ou intimider les comédiens. Au départ, nous étions une trentaine de personnes à travailler sur le spectacle; au final, nous nous sommes retrouvés à cinq, c'est pourquoi il n'y a que cinq personnages dans la pièce et plusieurs marionnettes pour incarner les comédiens partis - une astuce qui permit ainsi de poursuivre le travail malgré les nombreuses intimidations... On a eu beau faire le vide autour de moi, on n'est pas parvenu à m'intimider. Finalement, à la demande de l'ambassadeur de France d'alors, le directeur de l'Alliance française nous a demandé de quitter les lieux! C'était devenu compliqué pour moi de rester à Madagascar et, heureusement, la chance - ou la destinée - est intervenue au moment même où ma pièce était interdite... Quelques mois plus tôt en effet, j'avais adressé l'une de mes nouvelles au concours de Radio France internationale. Elle a été primée et j'ai pu ainsi bénéficier d'une bourse d'études d'une année en France. Aussi bien les personnes qui m'appréciaient que celles qui ne m'aimaient guère m'ont dit de partir. Si je voulais continuer à écrire, il fallait que je quitte le pays. Je suis donc parti. C'est à ce moment-là que j'ai ressenti ce choc entre ma conception du théâtre et ce qu'on entend, en France, par théâtre francophone. Je n'ai pas aimé et, en réaction, j'ai écrit des nouvelles qui ne sont cependant pas étrangères à ma conception du théâtre : le théâtre comme une mise en voix de l'être - expression que je préfère à " mise en scène " -, d'un souffle, une mise en voix des rapports humains qui s'accompagne d'une projection vers un imaginaire, vers un monde très vaste qui peut échapper aux comédiens, ou aux metteurs en scène en général. C'est pour cela aussi que l'on trouve des images, de la vidéo, de la musique dans mon théâtre. 
JULIE PEgHINI - Tu as écrit des poèmes en malgache...

Raharimanana - J'ai publié en effet un recueil de poèmes en malgache, j'ai aussi écrit beaucoup de chansons pour des groupes de Madagascar, en particulier pour le musicien Tao Ravao. Chez certains groupes, on ne trouve pas forcément de paroles ou de refrains au sens où on l'entend habituellement, pourtant ils partent toujours du verbe. C'est une sorte de musique de possession et la voix n'est pas immédiatement, disons, explicite, parce que le format de cinq ou même de dix minutes ne convient pas pour faire émerger les paroles. Il faut jouer toute la journée et, à ce moment-là seulement, la parole arrive. Au cours des répétitions, tandis qu'ils improvisent, je discute beaucoup avec les musiciens. Ils composent à partir des textes que je leur donne, et j'accepte qu'au final il n'y ait pas forcément tous mes mots dans leur musique. C'est avant tout une pratique d'écriture pour moi et le public n'est pas forcément au courant que je suis bien derrière tout ça.

Je voudrais aussi parler du recueil de poèmes Empreintes.

Empreintes, Vents d'ailleurs, 2015, p. 65

"L'empreinte est ce qui survit à la conscience »

(c) Vents d'ailleurs

L'empreinte est ce qui survit à la conscience, la mienne me brûle, ma peau est de trop, près de ma vie. Tout contact m'est pénible, le sais-tu, le comprends-tu, ce que je traverse encore aujourd'hui, entre cri et silence, ce cri dit hors raison, ce cri dit hors pensée, ce cri dit hors sens, je me tais, essayant de reconstituer ce qui fut gommé, rayé, biffé. Et sortant de mon silence, je bégaie, tu ris. Mais je sais pourquoi je bégaie, je sais pourquoi je crie. Ne l'attribue pas à la nature. Ne l'attribue pas à ma différence. Cri au-delà de toute langue. Cri car seule expression qui surpasse tout code, toute culture. Crier, c'est venir à toi par le corps, et nous prévenir des détours et dénis des raisonnements.

Un ami chorégraphe d'origine martiniquaise, Miguel Nosibor, m'a demandé de l'accompagner dans son travail : il voulait "reprendre mémoire ». Il avait lu mes livres où justement je parle souvent de reprendre mémoire. Formé à la danse de rue en France, Miguel cherchait à reprendre mémoire dans son corps, dans sa danse. C'est en discutant ensemble qu'il a commencé à refaire sa danse. Pendant deux ans, nous avons ainsi travaillé tous les deux, il dansait, j'écrivais et je lui donnais les textes...

Lecture d'un passage d'Empreintes par Raharimanana accompagné de la valiha, p. 32-33

je te pose à mon écoute tu n'as pas à bouger je te pose à mon écoute tu n'as pas à dire je te pose à mon écoute 
tu n'as pas à t'élancer pour m'aider et dénoncer m'aider et lutter m'aider et réparer m'aider à faire souvent

revenir

souvenirs mémoire histoire mythe légende je te pose à mon écoute

tu n'as pas à parler tu n'as pas à répondre tu n'as pas à accepter tu n'as pas à résister tu n'as pas à... argumenter

je te pose à mon écoute car de réparation je n'en voudrais que pour mes morts qui n'en jouiront jamais

on ne répare pas les morts les mots sont morts

de mémoire il m'en manque il t'en manque

je te parlerai sur du vide et de l'extermination je te parlerai sur ce que je ne sais pas mais que je ressens profondément dans ma chair je parlerai sur ce que je ne sais pas sur ce que tu ne sais pas et que tu ne pourras jamais ressentir ce ne sera pas raisonnable car les sens ne se comprennent qu'à la folie

il te sera presque impossible de m'écouter car cette intelligence n'est pas la tienne il te sera presque impossible de m'écouter car ce temps n'est pas le tien et tu ne conçois pas que j'y vive moi qui suis à côté de toi.

on ne répare pas les morts sont morts

et pour me guérir il faut m'effacer et t'effacer et nous effacer il nous faut disparaître avec ce mal effacer jusqu'à la moindre empreinte

je te pose où je ne veux pas je te pose où tu ne veux pas à l'écoute qui nous consume mon ami mon ennemi mon frère mon sauveur mon bourreau

tu es le fils de qui je suis le fils et qui nous as engendrés monstrueux d'ignorance la cruelle domination

C'est difficile à expliquer mais il y a ici comme une sorte de prise de possession du Verbe. Je travaille beaucoup sur ce que l'on appelle le tromba à Madagascar. Tromba c'est le Verbe qui descend et qui va habiter le corps. Le corps se donne totalement au Verbe, il n'a aucun contrôle sur lui car celui-ci décide de tout. La personne qui est possédée doit être dans ce mouvement-là, du Verbe. J'adopte ce procédé dans mon écriture, l'auteur, finalement, est à disposition entière du Verbe et accepte le noncontrôle. C'est pour cela que l'écriture n'est pas fixe et que la partition est absurde. Le mouvement doit arriver, les émotions doivent être les notes. Cette écriture doit être investie par le Verbe. C'est la raison pour laquelle je n'ai pas ponctué ce texte; je cherchais à faire ressortir la fluidité de la parole orale, le souffle qui n'a pas la rigueur de la ponctuation.

Entre la poésie et le théâtre, j'opère beaucoup de connexions, ce qui n'est pas facile car les comédiens ont une culture différente, entre autres déjà celle d'apprendre le texte par cœur. Or, dès qu'un comédien connaît un texte par cœur, cela lui devient difficile de s'en détacher, de se détacher d'une certaine diction comme on peut la trouver dans les écoles de théâtre. Quelque part, je demande qu'après la possession du texte, il faut s'en déposséder, et ne pas se focaliser sur le sens, accepter que dans la parole passe aussi autre chose d'indéfinissable.

Ce genre de dire que je recherche, nous le retrouvons dans les cérémonies malgaches qui ne sont pas des spectacles que l'on écoute, que l'on regarde. Non, il s'y passe autre chose, car il n'y a pas vraiment de public : chacun est dedans, chacun entre, d'une certaine manière, dans la cérémonie. Bien évidemment, le Verbe ne descend pas en tout le monde, mais si vous avez envie de taper des mains, vous pouvez le faire. Si le Verbe vient à vous, vous rentrez alors dans le Verbe. Ce n'est pas aussi normé qu'une pièce de théâtre. Peut-être arriverai-je un jour à faire ce genre de théâtre. Avec des musiciens je peux y parvenir, mais avec des comédiens, ça m'est difficile, sans parler 
surtout de la configuration des salles de théâtre où une coupure existe entre le public et l'espace scénique.

NiColAS MARTIN-GRANel - Peut-on dire que le roman Za part du genre oratoire qu'est le kabary?

Raharimanana - Dans $Z a$, j'ai beaucoup travaillé aussi l'ancien français. Le kabary fait partie bien sûr de ce roman, mais ce n'est pas que ça. À Madagascar, on ne peut pas se proclamer maitre de la Parole comme cela, la Parole, en fait, ne peut pas avoir de maitre, car la Parole est au-dessus de tout, il n'y a que les morts qui peuvent s'en prévaloir, et encore ! Ça dépend du mort ! On ne peut s'ériger ainsi en maître de la Parole car celle-ci échappe à tous. Elle est au-dessus des vivants et appartient aux ancêtres. Lorsque vous prenez la parole, il y a toujours une faute quelque part. En effet, quelle est votre légitimité ? Peut-être y a-t-il d'autres personnes plus aptes que vous à saisir cette parole? C'est pourquoi on commence par s'excuser pour toutes les éventuelles maladresses que l'on pourrait commettre. Mon roman $Z a$ débute par les «Excuses et dires liminaires de Za». Je m'inspire bien ici de cette forme traditionnelle malgache, très codée et noble, qu'est le kabary ("liminaires", quant à lui, renvoyant au titre d'un recueil d'Aimé Césaire.) Le kabary, c'est de la poésie pure, l'usage de métaphores et de belles formules, l'emploi d'un langage empreint de précaution. Mais je dévergonde complètement cette forme, sans pour autant m'en moquer, en lui greffant la langue du sôva, un autre genre de poésie traditionnelle malgache fait, disons, pour se moquer des gens: celui qui parle doit donner l'impression d'être fou, ivre mort, inconscient des propos qu'il tient. Je prends donc ce langage-là et j'invente autre chose. J'opère un va-et-vient entre les genres, je cherche à approcher la complexité de l'oralité à travers l'écriture d'un roman de littérature française.

JULIE PEgHINI - Dans un entretien, tu as dit que le discours est proprement monstrueux, ce qui me fait songer à Machin la Hernie, de Sony Labou Tansi, à cette idée du monstre en soi, du langage comme un monstre...

Raharimanana - Le monstre a une fonction, celle de nous confronter à nos réalités. Voir le monstre c'est comprendre que tout est susceptible d'être anéanti. Mais le monstre c'est aussi autre chose que l'on n'ose pas aborder. Dans $Z a$, je me suis persuadé que rien ne devait me faire peur lorsque je commençais une phrase. Ce n'est pas une folie de se dire que l'on peut être remis en question, que le langage même n'est qu'une petite manifestation de toutes les possibilités d'être, de s'exprimer... Je me suis fixé une règle très simple concernant $Z a$ : je prenais les deux langues, la langue malgache et la langue française, et je comparais. Au niveau de la prononciation ou plutôt de la maîtrise, je dirais physique des sons, je me suis interrogé sur les difficultés de prononciation d'un Malgache ou d'un Français. Un Malgache va éprouver du mal à dire le mot « jugement », par exemple, car il n'y a pas de [u] dans la langue malgache, il n'y a pas de [j], il n'y a pas de [œ], et le [mB] se prononce [ma]. C'est pourquoi dans $Z a$, toutes les chuintantes se transforment en [z], les [u] en [i], le [œ], lui, peut se prononcer [a] ou [e].

Je me suis imposé une autre règle relative cette fois à la syntaxe française où l'on trouve la construction : sujet-verbe-complément. En malgache, c'est différent, on a le verbe, le complément et éventuellement un sujet. Lorsque j'apprenais la langue française, j'avais peur de parler car je devais commencer par «je», ce qui est très malpoli chez nous. C'est pourquoi j'ai transformé «je » par za (za signifiant « moi » en 
malgache), et je l'ai conjugué à la troisième personne du singulier, ce qui a complètement bousculé la langue française. Lorsqu'il y a des chuintantes, Za zézaye. Comme les sons se transforment, le sens aussi bouge et conduit à d'autres sens. Le monstre se situe ici. Ce que vous teniez comme absolument inébranlable peut subitement s'effondrer. La langue française académique, qui n'est finalement qu'une construction, peut s'effondrer.

JULIE PEghiNı - Pourrais-tu nous lire un passage de ton dernier livre, Revenir, avant que nous en parlions ensemble?

NiColAS MARTIN-GRANé - Avant que Jean-Luc ne débute sa lecture, je voudrais signaler qu'un thème commun traverse son dernier livre et celui de Sami Tchak, celui de la figure du père. Dans la rentrée romanesque de cette année, ils ne sont pas les seuls d'ailleurs à avoir fait appel à cette figure. Je peux citer Empreintes de crabe de Patrice Nganang, /l est déjà demain d'Henri Lopes, Camarade papa de Gauz ou encore Un si beau diplôme de Scholastique Mukasonga, récit autobiographique qui rend aussi hommage à son père.

Lecture d'un extrait de Revenir par Raharimanana accompagné de la valiha.

Refuge de l'âme, quelques oublis, ou la divagation dans les pensées sans loi ni récit. Hira se réveille. Ou peut-être qu'il rêve. Quelle importance lorsqu'on dort sur un lit de sable? Le fleuve scintille malgré la nuit. Le pont n'est visible que par son ombre. Les étoiles ne se noient pas contrairement au regard de Hira. Ce qu'il voit ne l'étonne pas. Du pont, les martyrs, un à un, prennent leur envol. Hira les regarde sans réagir. Jamais n'est de noir sur les rives des fleuves, l'eau est miroir qui éclaire. Les martyrs volent comme les oiseaux. Hira se relève de son lit de sable, il prend le vent léger qui souffle sur sa joue et lui aussi s'envole, lentement, torpillé dans le dos par les grains de sable qui regrettent le poids de son corps. Il s'appuie sur le vent léger et rejoint ses compagnons qui flottent au-dessus du pont. Ainsi donc, vous avez été massacrés ici par un énième dictateur...

Oui, disent les martyrs du pont, allons-nous-en!

Hira.

Vent doux et sa propre lourdeur.

Hira

La lourdeur n'est pas de son corps, elle est de sa rage.

Vent doux.

Hira quitte une terre et s'apprête à se rabaisser sur d'autres sols qui ne le reconnaîtront qu'exilé, étranger.

... vole!

Chant

Hira est un chant.

Dites-moi quelle est la couleur de la nuit quand elle meurt.

Hira voit son corps tout en bas. Il entend les cris de bien d'autres hommes, femmes, allongés parmi les balles perdues du pont des Martyrs.

Allons-nous-en, lui redisent les martyrs du pont.

Hira ne se penche plus vers la terre, il ne se retourne plus. Il entend les cris souhaitant un bon voyage, bon courage, belle Révolution. Les cris sont dispersés par le vent et fouettent les drôles d'oiseaux qu'ils sont : ô la nuit, les oiseaux qui de basse lune fuient le tumulte des hommes, je suis de vous.

La désespérance, forme ultime de la lucidité, Hira garde les yeux ouverts et entre en écœurement.

JULIE PEgHINI - Pour refaire le lien avec le théâtre, je repense à ton texte Parfois le vide, avec ces gobeurs d'étoiles qui sont comme des martyrs.

Raharimanana - Parfois le vide est un spectacle que je viens de donner et où l'on retrouve ce que j'entends par gobeurs d'étoiles. On rêve, on gobe les étoiles. Mais c'est lié aussi à un conte malgache où des enfants demandent toutes sortes de choses à leur père et, comme celui-ci est un géant, il peut tout avoir, tout attraper. Puis, les 
enfants veulent manger, gober les étoiles, alors le père commence à saisir les étoiles. Il y en a tellement dans le ciel, ce n'est pas bien grave! Pour finir, les enfants demandent à gober la Lune. Se pose ici un problème : il n'y a qu'une seule Lune. Le conte finit ainsi, sans que l'on sache ce qui va se passer et c'est ce qui le rend extraordinaire. Chaque conteur pourra inventer une suite qui saura expliquer, peutêtre, le pourquoi de la face cachée de la Lune. À travers ce conte, on comprend qu'on invente tout.

Écrire est fabuleux car vous n'êtes pas obligé d'étaler votre culture ou de justifier, expliciter... Et puis on a droit à l'hermétisme qui n'est pas un enfermement mais bien une porte ouverte sur le rêve.

Pour revenir à la lecture de cet extrait, c'est à Bamako que je l'ai écrit, sur un pont qui m'a de suite attiré, le pont des Martyrs. Une nuit, je m'y suis rendu et je suis resté là, à regarder. Ce fut à la fois une sorte d'expérimentation personnelle, d'imprégnation de la nature et de voyage empathique. Je ne savais pas pourquoi on appelait ce pont «Le pont des Martyrs ", et c'est plus tard que j'ai appris ce qui s'y était passé.

Projection d'un extrait vidéo du spectacle Empreintes

https://vimeo.com/112476027

Raharimanana - Ici, vous entendez des sons que j'ai captés, ainsi que le chant de possession d'une artiste malgache, Mama Sana. C'est l'ingénieur du son qui a réalisé la bande-son selon mes indications.

Fin de la projection

Raharimanana - On parle beaucoup de l'éclatement des genres; pourtant, me concernant, on aborde mal les choses. Est-ce que je vais faire de la poésie, du théâtre, du roman? Au moment même où j'écris, je ne me pose pas du tout la question du genre. J'écris selon comment je perçois, comment je reçois le verbe. À ce moment-là, je peux me trouver sur scène avec un musicien, un artiste, ou même face à des photographies. Ensuite, cet écrit pourra devenir un roman, un essai, de la poésie, du théâtre, dans l'absolu je n'y accorde pas beaucoup d'importance. Cela dit, il y a les règles du marché éditorial!

Pour en revenir au théâtre, une chose est certaine, je n'ai pas encore fait le théâtre que je veux, juste partiellement.

Dans la vidéo que vous venez de visionner, notre principe, à Miguel Nosibor et moimême, était de construire ensemble le spectacle au fil de notre musique et de réinterroger complètement l'espace scénique - l'architecture du théâtre -, la place du public, ainsi que la mentalité même du spectateur. S'il devait y avoir des réactions dans la salle, pourquoi un spectateur dirait-il «chut »? Le public ne doit pas être timide. Le théâtre peut être une aventure inachevée.

NICOLAS MARTIN-GRANEL - Tu parlais de photographies. Peux-tu revenir sur ton expérience de commissaire d'expositions, sur ce travail de quasi-historien, anthropologue que tu as opéré au cours de ton enquête de terrain avec Pierrot Men à Madagascar?

INTERVENANT - J'ouvre une brève parenthèse en lien avec ce que tu disais sur le théâtre. Platon dit une chose terrible sur le théâtre grec, un théâtre qui, dans toute son histoire, a toujours été l'occasion de l'invective, de l'expression, de l'échange en doublant la vie de tous les jours, entre drame et tragédie. II est intéressant de constater l'indignation de Platon, ce qu'elle construit et l'héritage que l'on en a aujourd'hui.

NICOLAS MARTIN-GRANEL - $\|$ faut exclure les poètes de la cité... 
INTERVENANT - En effet, tous ceux qui vocifèrent, qui sont la vie même, et qui ont besoin de ce lieu pour s'exprimer.

Raharimanana - Avant de revenir à 47, je vais aborder mon rapport à la photographie. Je ne sais pas si je fais de la photographie, toutefois elle fait partie de mon brouillon d'écriture. Giacometti se plaint de ne pouvoir fixer la marche ou peindre les paysages car ils changent sans cesse, ils sont mouvants. Ce constat de Giacometti m'accompagne lorsque je regarde le monde où tout change effectivement à chaque instant. Avec la photographie, il y a cette possibilité de fixer l'instant, de capter mes visions, c'est-à-dire des images qui me parviennent à des moments très précis. Lorsque nous regardons quelque chose, l'océan par exemple, qui n'a jamais cherché à voir un personnage ou des corps, ou même une tête, une bouche, un œil ?

On a subitement une vision et on prend alors la photo.

Pendant les répétitions d'Empreintes avec Miguel, à un moment j'ai regardé le sol et d'un seul coup, entre ses jambes, j'ai vu un oiseau.

Fig. 2 : L'albatros

Photo de l'auteur

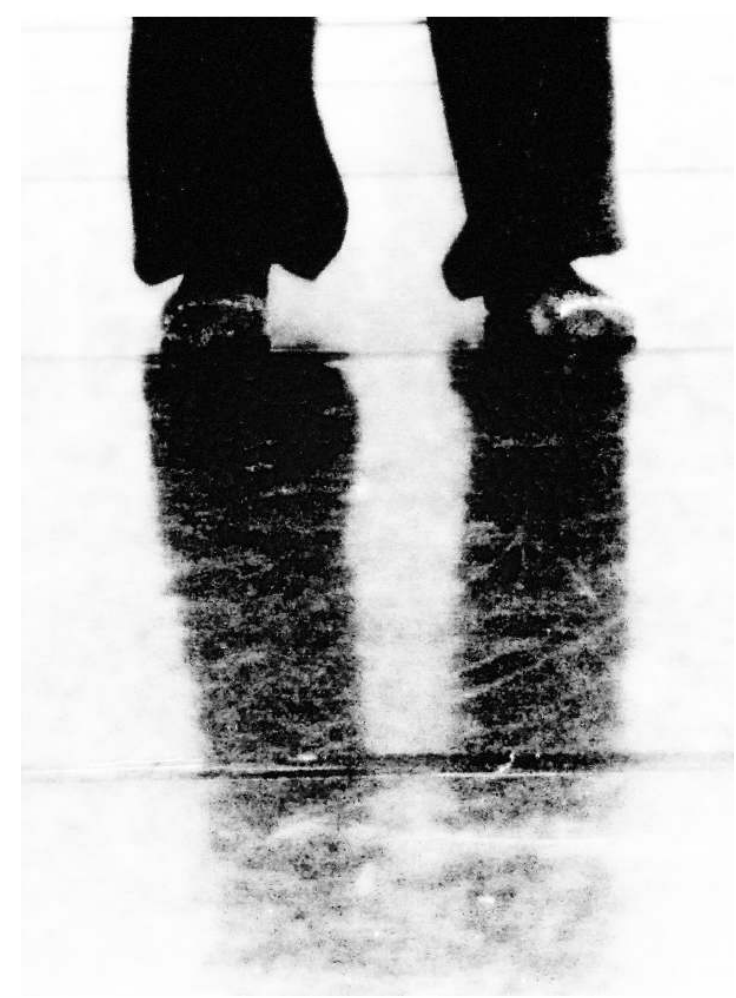

Dans l'extrait vidéo que je vous ai montré, si vous regardez bien l'ombre entre ses jambes, vous apercevrez cet oiseau. C'est à cet instant précis que j'ai pris la photo. Même chose pour les traces de chaussures où j'ai vu un masque africain.

Je ne fais pas de la photo pour être photographe. Un jour peut-être réaliserais-je une exposition, mais je ne peux pas affirmer que je suis un photographe même si ce médium est utilisé dans Le Cauchemar du gecko ou dans Empreintes, ou encore Des ruines. J'insiste : la photographie fait partie de mon brouillon d'écrivain.

Fig. 3 : Flux d'écorce

Photo de l'auteur

Continents manuscrits, 12 | 2019 


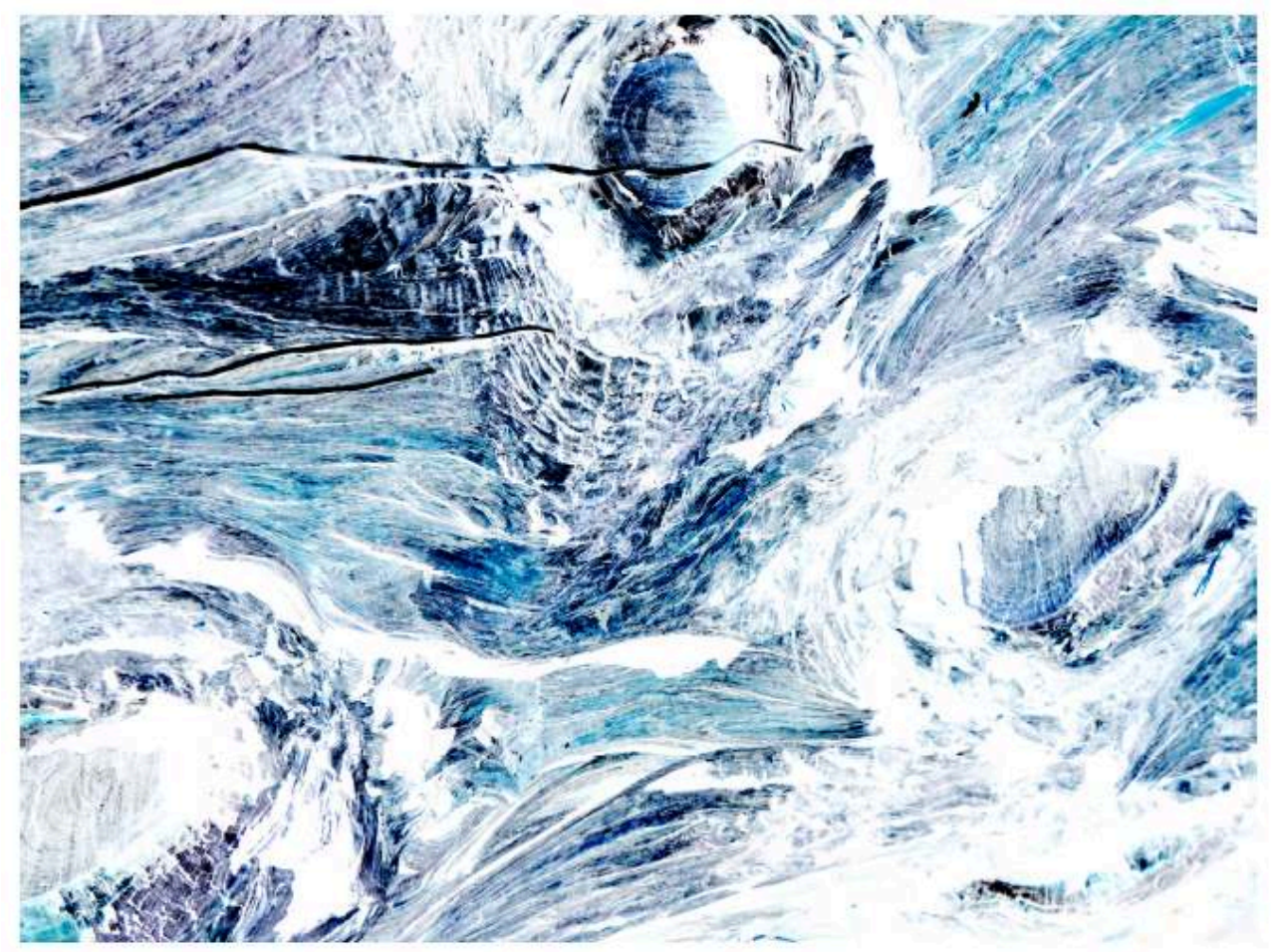

Fig.4 : Mavo

Photo de l'auteur

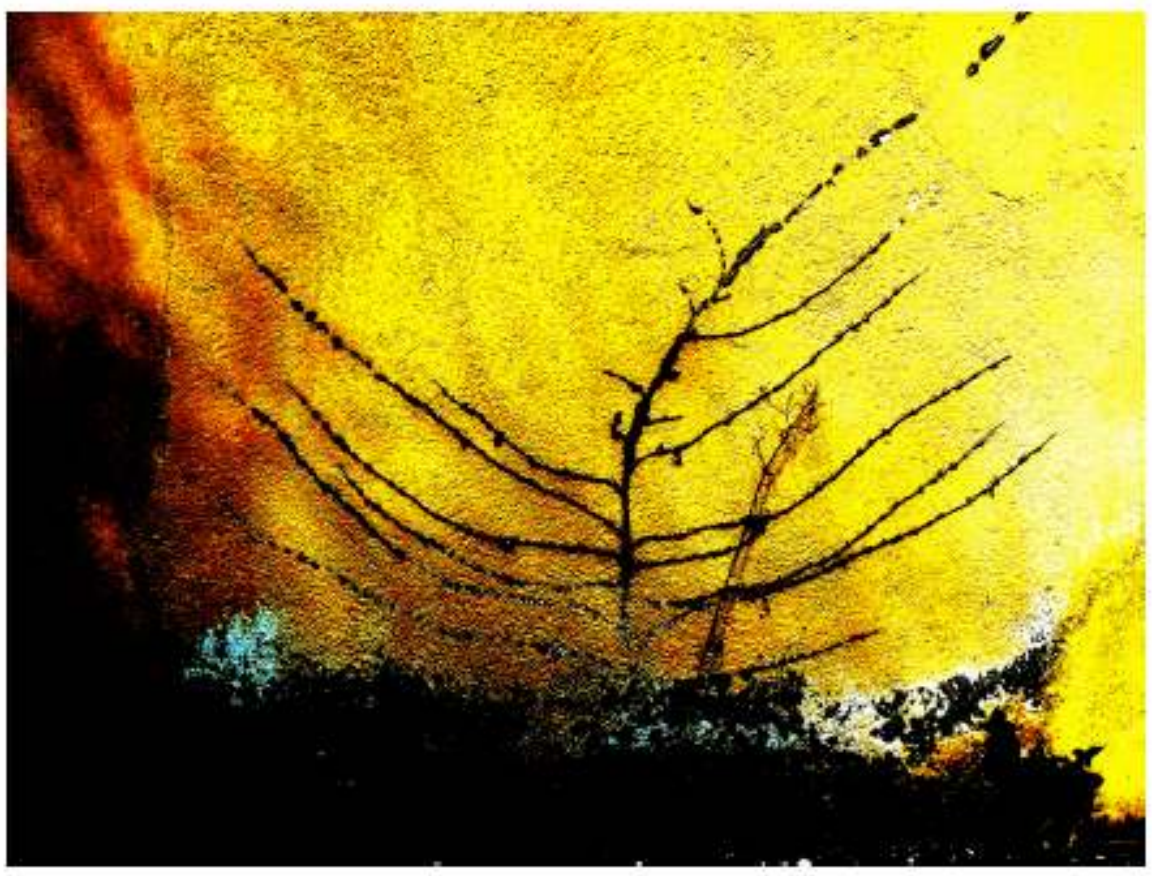

Fig. 5 : Empreintes (main)

Photo de l'auteur 


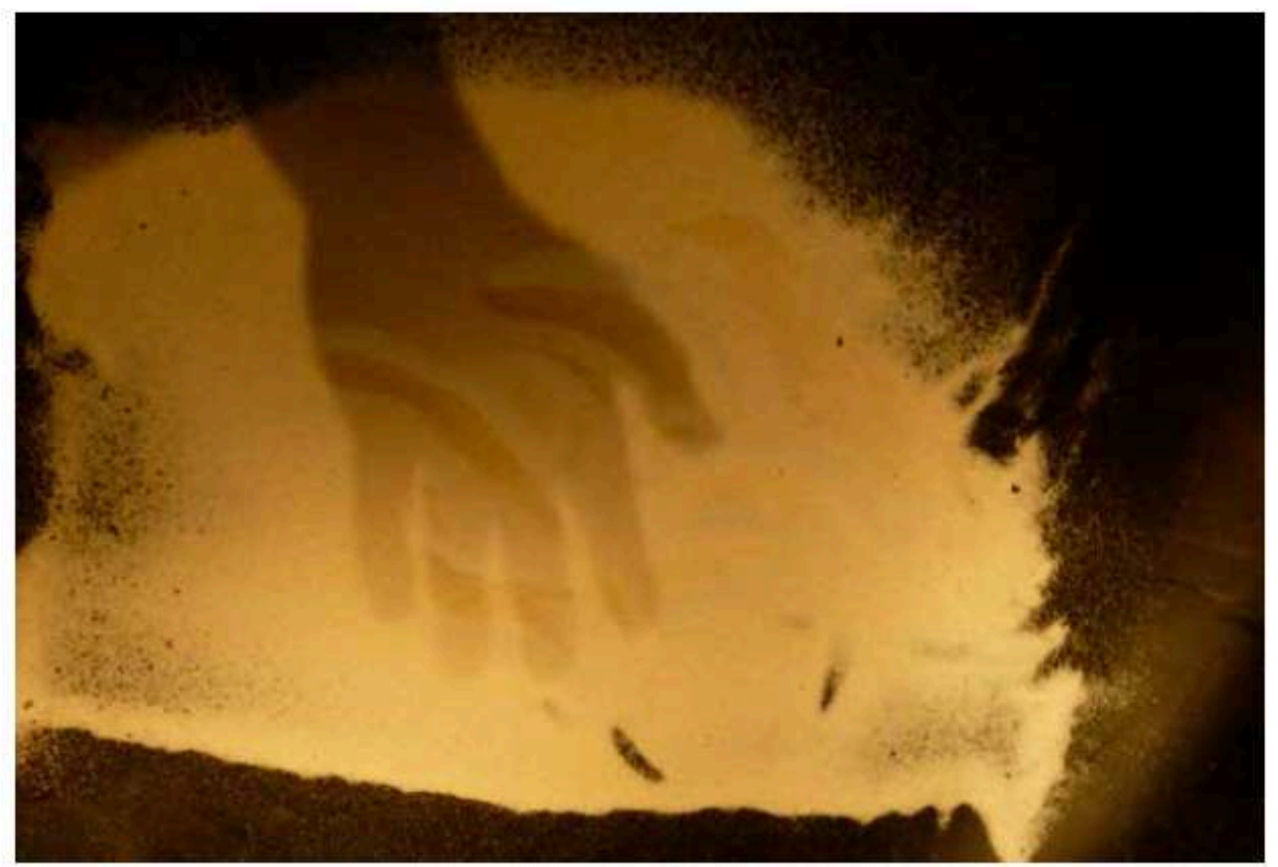

Fig. 6 : Empreintes (mains 2)

Photo de l'auteur

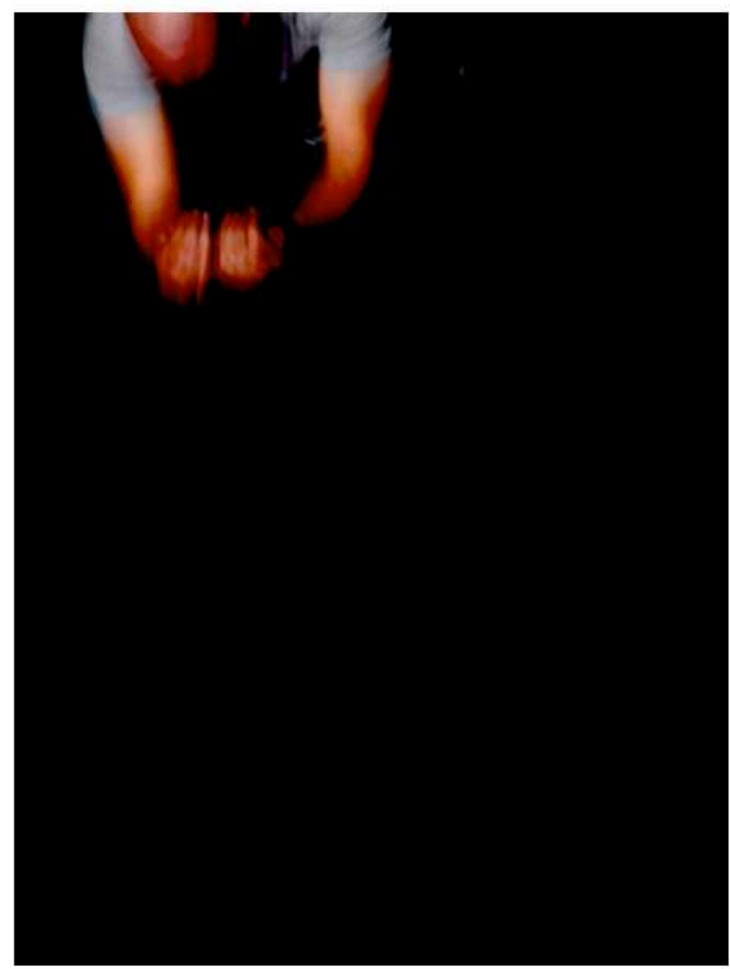

En 2001, j'ai publié Nour 47, un roman sur la rébellion et les massacres de 1947 à Madagascar. Le public malgache m'a découvert avec ce texte et il y a eu une véritable reconnaissance de sa part. Avec mes livres précédents, j'étais apprécié ou détesté, voire contesté. Les débats étaient passionnés et la polémique jamais loin. Sitôt la publication de Nour 47, j'ai reçu beaucoup de lettres de Malgaches me demandant de raconter 
encore, et j'ai écrit Madagascar 1947. Par la suite, Thierry Bedard a transformé ce texte en pièce de théâtre, 47. En 2008, nous devions opérer une tournée dans les centres culturels, instituts français. Le ministère des Affaires étrangères français, qui avait pourtant financé la production du spectacle, a finalement interdit cette tournée. Nous avons écrit à Bernard Kouchner, le ministre d'alors, mais pour couvrir le ministère celui-ci nous a signifié que les instituts étaient libres de programmer ou non cette pièce. Or, la pièce était déjà programmée dans ces lieux! La censure était donc bien réelle. Alors, quelle réponse donner à cela ? Une réponse polémique? Mais si j'allais sur ce terrain, la polémique étaient perdue d'avance. Je ne voulais pas, je ne veux pas affronter des personnes comme Zemmour, par exemple. Cela ne m'intéresse pas car ce n'est pas de la pensée, il n'y a pas de vrai. Ce genre de débat avilit.

Donc, comment répondre à cette censure? Eh bien, j'ai décidé de retourner à Madagascar. Les « vieux » sont en train de mourir, il faut qu'ils puissent raconter. J'en ai parlé à un grand photographe malgache, Pierrot Men. Je n'avais même pas terminé ma phrase qu'il a dit oui et, vingt-quatre heures après, il était déjà sur le terrain. Au début je n'ai pas bien compris sa précipitation à accepter ce travail. J'ai appris, par la suite, que sa famille avait été touchée aussi par les événements de 1947, pourtant il n'avait jamais osé aborder ce sujet dans ses photos. Certes, il avait pris des photos d'anciens combattants mais seulement lors des commémorations du 29 mars, comme un photographe officiel sans qu'il n'y ait rien de personnel dans son travail. Je vais vous montrer quelques-unes de ses photographies.

Projection de photographies

Je lui avais demandé aussi d'enregistrer les gens qu'il allait rencontrer et photographier, et c'est à partir de ces enregistrements que j'ai écrit Portraits d'insurgés, la pièce Rano Rano et réalisé l'exposition présentée à Avignon dont voici quelques photos.

Fig. 7 : Martial Korambel, exposition "Portraits d'insurgés"

Photo de Pierrot Men

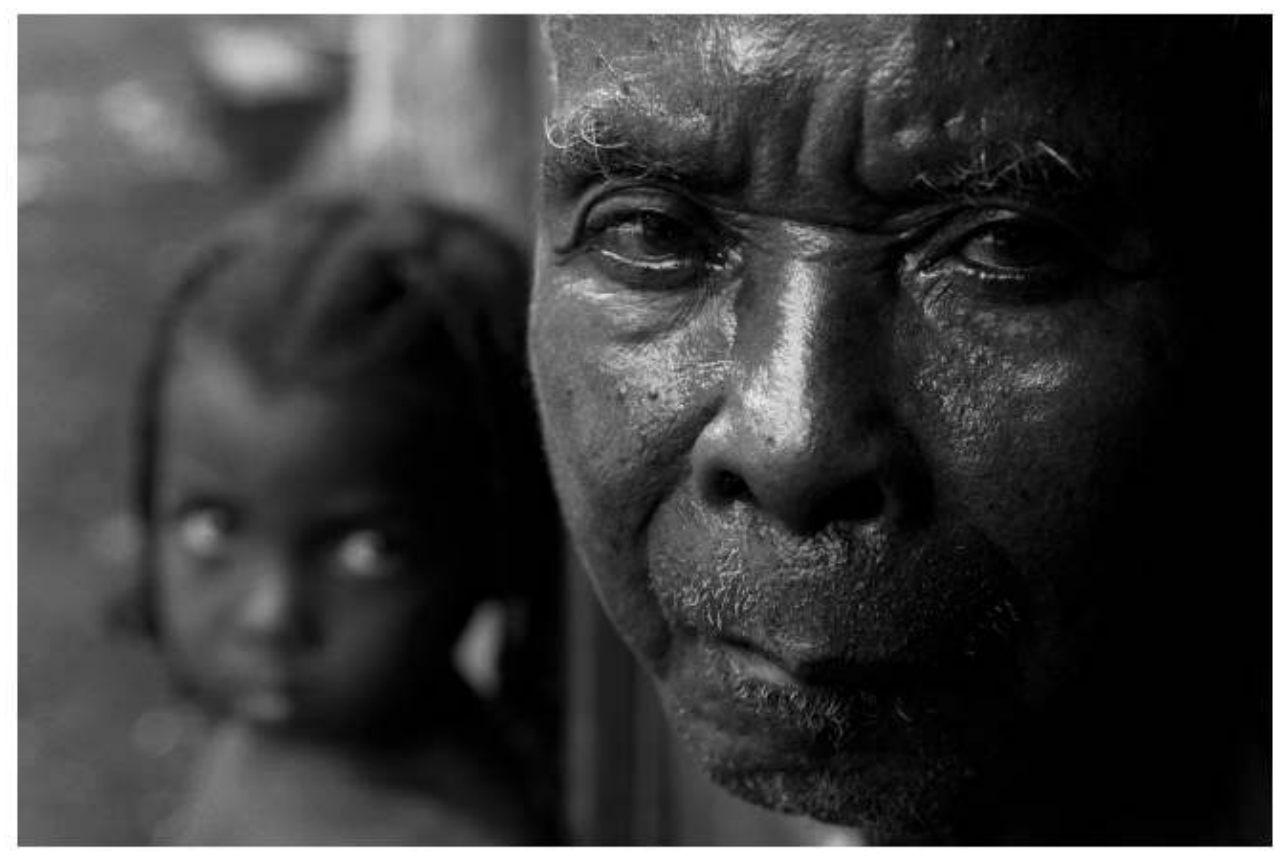

Continents manuscrits, 12 | 2019 
Fig. 8 : André Ralaivao, "Portraits d'insurgés"

Photo de Pierrot Men

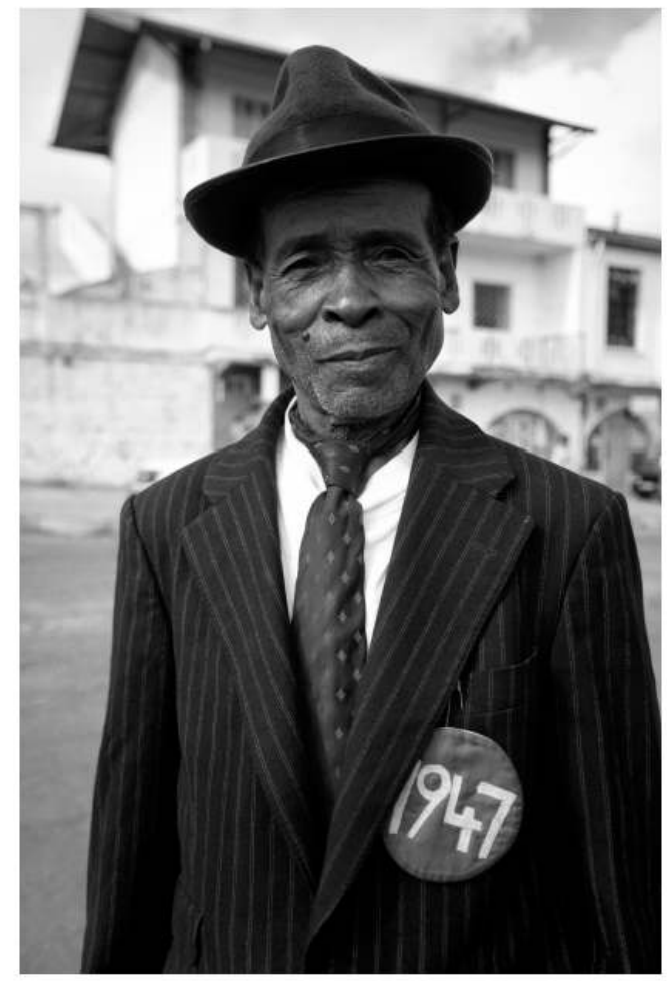

Fig. 9 : Extrait de "Portraits d'insurgés"

Photo de Pierrot Men 


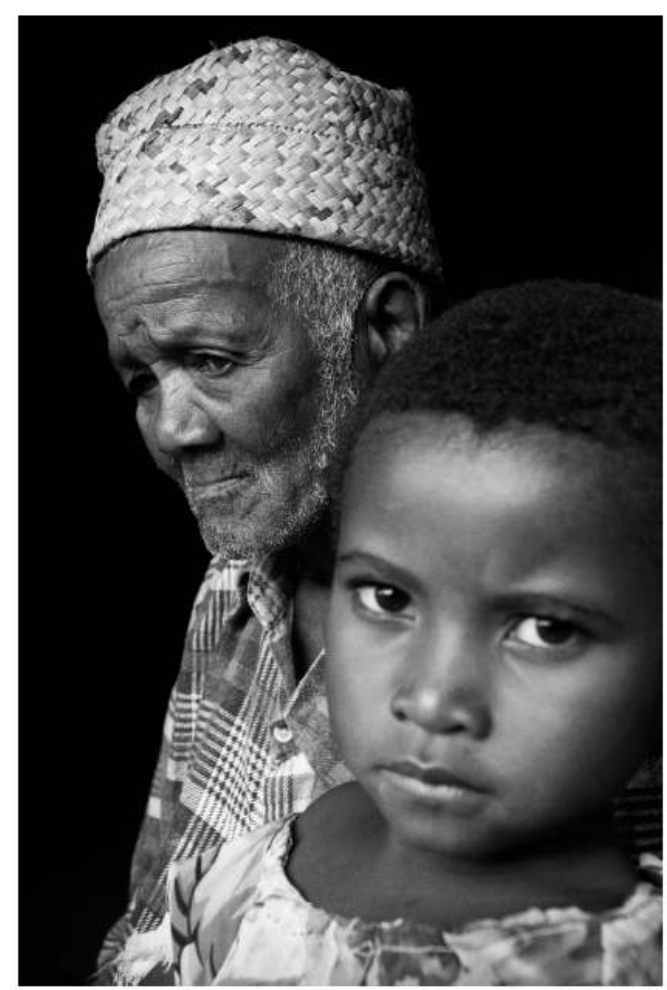

Fig. 10 : Philippe Ralison, “Portraits d'insurgés”

Photo de Pierrot Men

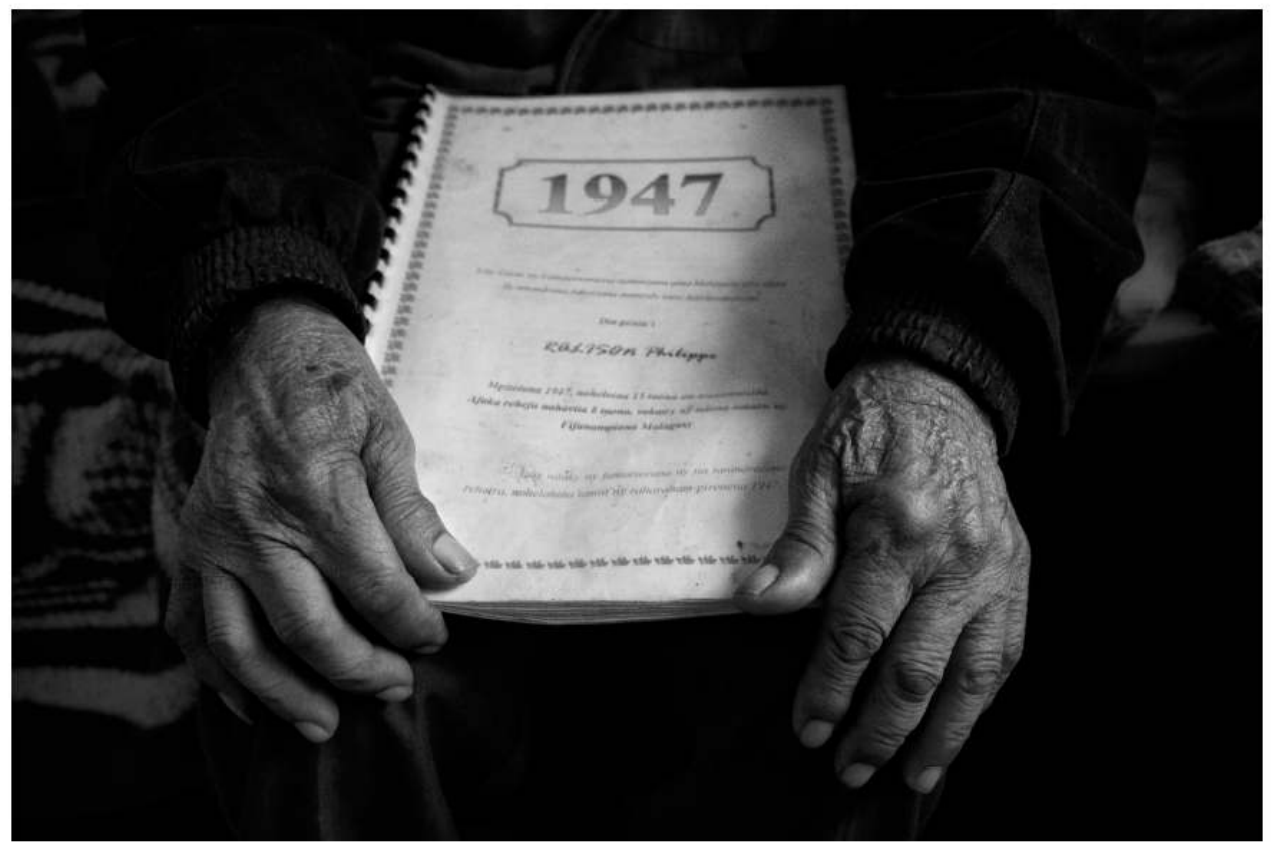

Fig. 11 : Zacharie Rafetison, "Portraits d'insurgés"

Photo de Pierrot Men 


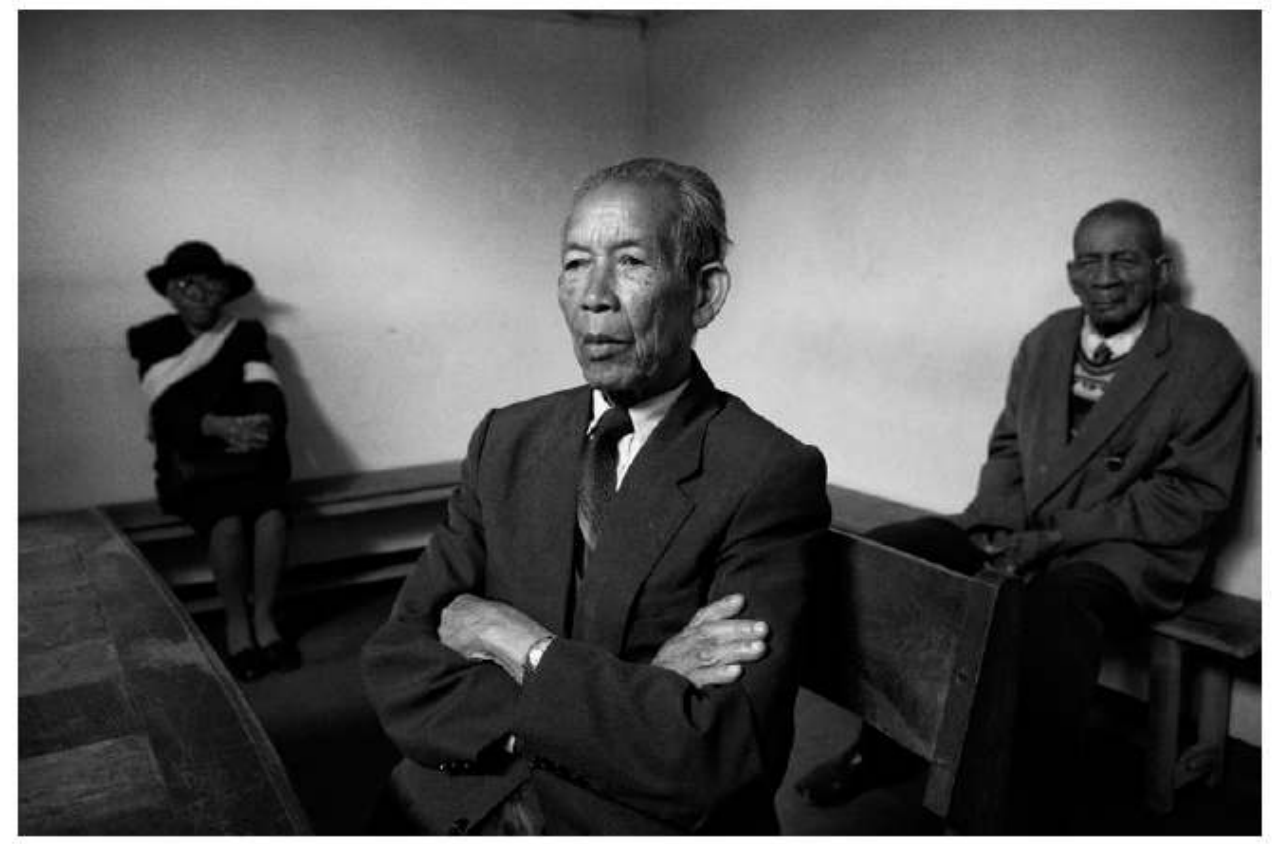

Fig. 12 : Emmanuel Ralahy, “Portraits d'insurgés”

Photo de Pierrot Men

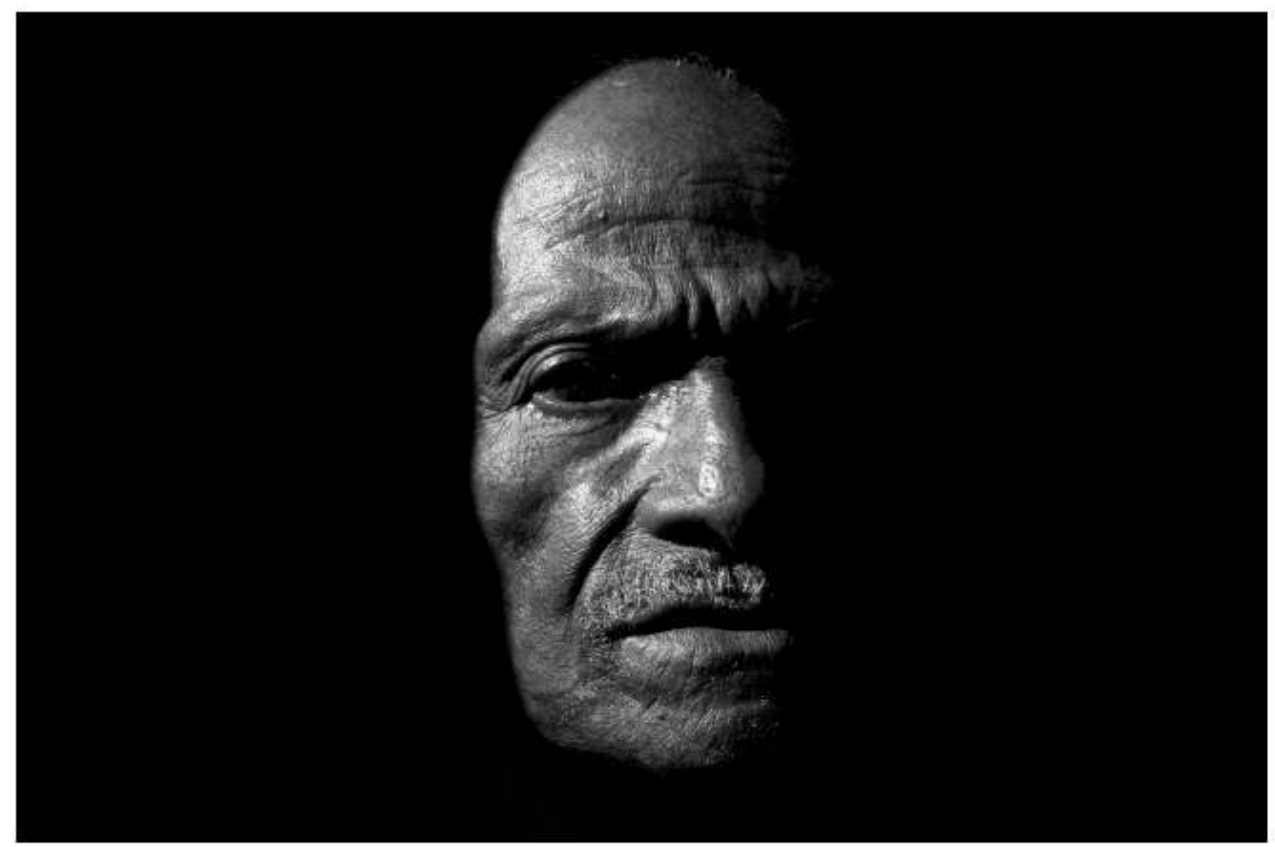

Fig. 13 : Felix Robson, "Portraits d'insurgés”

Photo de Pierrot Men 


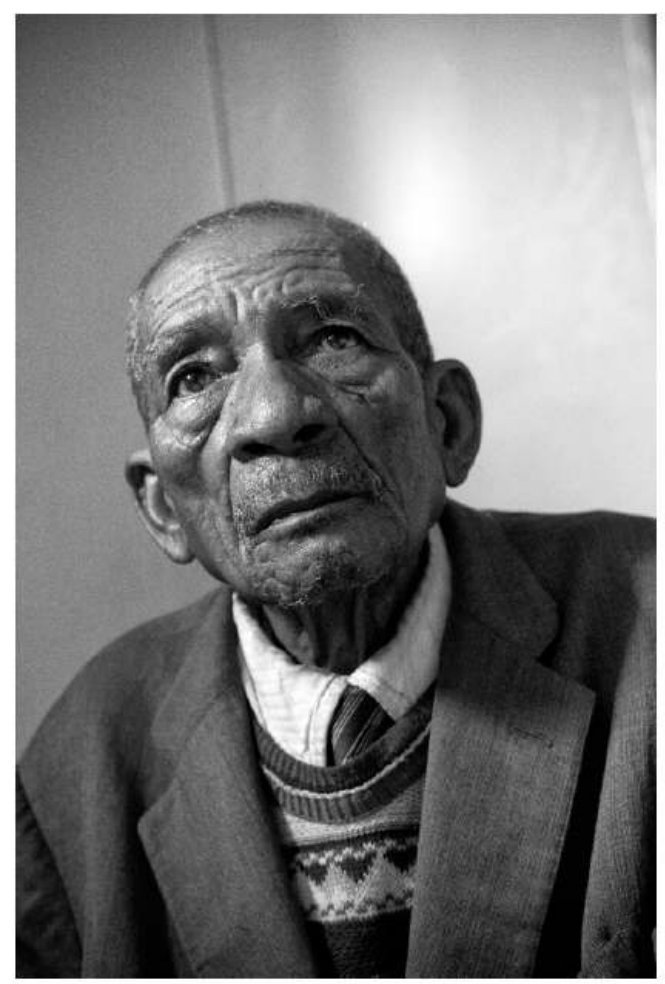

NICOLAS MARTIN-GRANEL - Tu as traduit en français ce que t'ont raconté ces hommes. Une photo en particulier m'avait frappé, celle où l'on voit ce vieux qui dit: "Tu parles une langue qui me fait mal parce que la langue est traumatisante. » Est-ce à toi qu'il s'adressait parce que tu lui parlais en français?

Raharimanana - Je lui parlais en malgache bien sûr. Mais j'écris et publie en français, et cela lui faisait mal : qu'un Malgache écrive en français et soit une figure de la francophonie.

Les trois personnes âgées que vous voyez sur cette photographie (fig. 11) sont très bien habillées. L'un des vieux, qui a vécu des choses très compliquées, a beaucoup raconté, et ce dès les années 1960. La dame que l'on voit à côté de lui a été torturée mais elle ne voulait pas témoigner. L'autre monsieur, lui, ne racontait que des bribes; il cherchait d'abord à comprendre qui se trouvait en face de lui. Que voulait cette personne vraiment? Pour en faire quoi, des témoignages recueillis?

Sur cette même photographie, vous avez Zacharie Rafetison, le président des anciens combattants malgaches, qu'il ne faut pas confondre avec les anciens tirailleurs! Il s'agit d'anciens rebelles, reconnus en tant que rebelles. À Madagascar, on trouve en effet des personnes qui veulent profiter du statut d'ancien combattant et qui s'inventent donc un passé de rebelles. Depuis 1980, l'État malgache alloue une allocation aux anciens combattants (une somme qui n'a quasiment pas bougé en presque quarante ans). Il existe donc toute une série de mesures permettant de prouver que telle ou telle personne est bien un ancien combattant rebelle.

Je reviens à la photographie. On remarque une petite table à côté de lui, avec deux tiroirs pour mettre les pensions des anciens combattants du district qu'il gère.

En 47 , cet homme, le président, s'est retrouvé dans une prison, où, m'a-t-il raconté, ils ont été entassés comme des animaux, les gens se trouvaient les uns sur les autres, les corps des uns sur les corps des autres, et lui, il était tout en bas, il respirait la merde. Il nous expliqua que c'est ainsi qu'il n'a pas eu à respirer le gaz, car les Français ont 
projeté du gaz pour réduire le nombre des prisonniers. Ce n'était pas la chambre à gaz des Nazis, mais ils ont quand même lancé du gaz... Lorsqu'il racontait ainsi, il fallait qu'il retrouve sa dignité, d'où l'élégance de son habillement et d'où aussi la nécessité de savoir qui se trouvait en face de lui. Est-ce que cette personne était digne de recevoir sa parole, son témoignage? Saurait-elle les respecter? De même, il cherchait à savoir si cette personne portait de la haine en elle. Si c'était le cas, il ne prendrait pas la peine de raconter. Il a été le plus réticent à parler, car j'écrivais en français. Pour lui, j'étais d'abord quelqu'un qui appartenait à la France, entre autres parce que l'on me voyait souvent à l'Institut français! Puis, notre "guide » ou «intermédiaire», une jeune femme qui n'apparaît pas dans cette photo, s'est énervée : lui qui se plaignait toujours que personne ne cherchât à raconter leur histoire se défaussait maintenant devant quelqu'un qui avait pourtant fait le voyage depuis la France pour recueillir son témoignage. Elle l'a littéralement engueulé, comme vous voyez, ces scènes où les petites-filles engueulent leurs grands-pères! C'est alors, plus tard, quand je me suis retrouvé seul avec lui, qu'il m'a montré le contenu du tiroir. Dans le tiroir, il y avait toutes les pensions des " anciens combattants ", des misérables billets qui ne suffisaient même pas à payer leurs transports de la capitale à leurs villages. Puis, il m'a conduit dans le couloir de la maison où les anciens combattants attendaient leurs pensions. Les anciens étaient assis le long des deux murs, et on passait entre eux. Tandis qu'on traversait le couloir, il leur expliqua que j'allais porter leurs paroles. C'est seulement après avoir quitté le couloir qu'il se décida à me confier son témoignage.

Après ça, il y a eu un projet de film auquel le président des anciens combattants a adhéré. J'ai fait appel à de jeunes cinéastes afin que la transmission de la mémoire s'opère. Dans cette pièce que vous voyez sur la photo, les anciens combattants ont défilé pour témoigner et nous les avons filmés. Au départ, nous devions être deux coréalisateurs: Marie-Clémence Andriamonta-Paes, qui est cinéaste, et moi-même. Mais le cinéma a une économie qui lui est propre. Moi qui n'avais jamais réalisé de film, je devenais un blocage pour le financement de cette production. Par ailleurs, mon passé disons polémiste compliquait encore davantage les choses. Enfin, et c'est important, Marie-Clémence, à cinquante ans, découvrait subitement que dans sa propre famille ils avaient été touchés par 1947 et que son propre père avait été emprisonné. C'est ainsi que le film est devenu son film, Fahavalo, Madagascar 1947. Cela m'a permis, non de passer à autre chose, mais de m'alléger un peu car, jusqu'alors, quand il était question de 1947, c'était toujours moi qui en parlais. Certaines personnes pensaient même que j'étais historien! Or, un tel événement ne peut être porté par une seule et même personne, ne refléter qu'un seul point de vue.

\section{Sami Tchak}

Sami Tchak - L'écrivain, qu'il parle des animaux, des arbres, des pierres, n'a qu'un sujet : la condition humaine; il est au cœur d'un anthropomorphisme. À quel moment un écrivain parvient-il à me toucher, quoi qu'il fasse subir à la langue ? À quel moment le travail de Jean-Luc Raharimanana me parle, moi qui ne connais rien aux codes malgaches? Si je ne parviens pas à entrer dans un texte, moi qui, dans ma singularité, porte la totalité de la condition humaine, alors l'écrivain ne me touche pas. Son matériau m'intéresse assez peu finalement, seul le résultat compte.

Jean-Luc expliquait que le nombre exact de victimes malgaches lors de la répression était nié. Mais heureusement qu'il existe des gens qui nient des vérités historiques 
établies, car c'est à ce moment-là que je perçois, chez un écrivain, ce qu'il a d'humanité. Lorsque l'on renvoie à quelqu'un l'image du monstre qui est en lui et que celui-ci dit que ce n'est pas lui, il réintègre alors une humanité dans laquelle je me reconnais. Ce n'est pas nécessairement une question juridique. À ce moment-là, le bourreau, ou le monstre, me devient sympathique car il réintègre ce que nous appelons les valeurs positives de notre société. L'histoire est faite de ces dénégations : quand le contexte historique change, nous ne voulons pas nous reconnaître dans ce qui fut peut-être un moment absolument jouissif ou légal, au moment même où on le vivait.

Jean-Luc n'est pas d'accord avec ma vision mais elle est essentielle. Le manichéisme facile, cette ligne de démarcation entre les bons et les mauvais, m'agace chez un écrivain, car il oublie qu'il parle de gens qui ont en eux une complexité humaine. Dostoïevski a la capacité de nous révéler l'intérieur de chaque personnage et sa complexité, qu'il soit le tueur ou le tué. C'est cette complexité que je demande à un écrivain, c'est pourquoi je parle de l'abeille et la mouche. Parvenir à la perfection de la vie d'une mouche n'est pas simple! Elle a beau patauger dans la merde, lorsqu'elle vient se poser près de ton lit, tu ne perçois aucune odeur. Le mot "mouche " ne pue pas.

J'ai une vraie fascination pour ce qu'on appelle les bas-fonds de la société. À Mexico, j'étais avec des gamins de la rue issus des favelas les plus cruelles de Bogotá - que je décris d'ailleurs dans Filles de Mexico. Quand je me rends dans ces endroits, je porte un fragment de ma mort, dont je n'ai pas peur, dans mon petit sac à main! Ces lieux sont absolument troublants, dangereux, ils ont une odeur, l'odeur de la vie. En tant qu'écrivain, c'est ce qui m'intéresse. Comment parvenir à en extraire ce qui parlerait le plus profondément à chacun de nous, nous des humains au cœur d'une même condition? La condition humaine, la condition du vivant. La mouche a ses plaisirs, ses maladies, ses doutes, des rêves que je ne connais pas mais que je peux imaginer. Peutêtre rêve-t-elle de voir l'un de nous transformé en cadavre... (Rires.) Toutefois, quel que soit son rêve, la mort, un jour, l'arrêtera. Tous les vivants ont cela en commun : la mort. $\mathrm{Au}$ cœur de toute littérature, je suis plus fasciné par la mort que par le théâtre luimême de la vie. Dans toute société, s'il y a une lumière qui irradie chaque individu, c'est peut-être celle de la mort. Cette conscience de la brièveté de notre passage donne un petit sens à notre vanité, à ce que nous disons, écrivons, ou à ce que nous croyons être.

INTERVENANT - Dans ce que vous dites, il n'y a pas de place pour l'hypocrisie. Je pensais à une maxime de La Rochefoucauld: "L'hypocrisie est un hommage que le vice rend à la vertu. » II y a des monstres qui prennent le masque d'humain et le manipulent; ils sont d'autant plus monstrueux et inhumains pour ça. Comment les percevez-vous?

Sami Tchak - Dans Ainsi parlait mon père, je parle de la place de l'hypocrisie comme l'un des acquis de notre civilisation. Si on privait la société de l'hypocrisie, je pense que beaucoup de nos fragiles équilibres s'effondreraient. L'hypocrisie est une nécessité : on l'utilise pour se conformer à quelque chose de beaucoup plus admis.

Je ne nie pas l'existence des monstres, j'ai même tendance à les préférer. Le monstre est la réalisation de beaucoup d'ombres communes aux êtres humains. Peut-être n'a-t-il pas nécessairement inventé ce qui le rend monstrueux. Nous n'avons pas tous le courage d'aller jusqu'au bout de ce qui nous passe par la tête ou au bout de l'ombre que nous portons dans nos cœurs. Il y a des monstres qui nous procurent de la jouissance : ce qu'ils ont fait, peut-être l'aurions-nous fait. L'horreur fascine les gens, mais nous n'avons pas tous suffisamment le courage d'atteindre un certain degré d'horreur. 
Quand je dis courage, c'est une provocation. Pourtant, si on regardait la proportion de personnes qui, un jour ou l'autre, ont rêvé de tuer quelqu'un, on trouverait des millions de personnes qui n'ont pas eu le courage d'aller jusqu'à tuer, mais l'envie ne leur manque pas. Nous portons tout de même en nous plus de désirs sombres que nous ne parvenons peut-être à les réaliser. Nous sommes civilisés pour nous contenir. Si la nuit nous garantissait un anonymat total, il y a beaucoup d'actes que nous commettrions. Le lendemain, nous montrerions un autre visage qui nous éloigne de la jouissance éprouvée à être l'homme de la nuit, ou la femme de la nuit !

Silence

RAHARIMANANA - Mais on porte aussi en nous des possibilités de lumière...

Sami Tchak - Oui, mais il y a des moments où elles ne sont pas nécessairement conformes à ce qui pourrait aboutir à une grande récompense. Il y a des lumières qu'on fuit parce qu'elles ne sont pas suffisamment récompensées. Dans le contexte malgache, si j'avais été à un tirailleur sénégalais, peut-être aurais-je trouvé une grande jouissance à massacrer des Malgaches. Puis, au moment où tu vas réaliser ton reportage, je ne le reconnaîtrai pas, parce que les temps auront changé. Il y a des moments où l'on agit sans avoir en tête l'idée de monstres, puisque c'est autorisé. Quand je travaillais sur les juifs, c'était la question qui se posait. Dans des villes comme Hanovre, lorsque l'on demande à un descendant d'Allemand nazi s'il éprouve de la honte ou du remords par rapport à ce qui s'est passé, la question est absurde, puisqu'il n'a pas été acteur. Or, si l'on posait cette question aux acteurs mêmes, certains diraient clairement que ce qu'ils ont éprouvé, c'était du bonheur. C'est ce qui m'a amené, d'ailleurs, à lire plus d'une fois Les Bienveillantes de Jonathan Littell. Si l'on avait garanti à des bourreaux qu'on ne les jugerait pas, je suis certain que l'on aurait entendu des descriptions de jouissance, ce que nous appelons aujourd'hui l'horreur. Mais qui, dans le contexte où ces hommes agissaient, était vraiment infect?

INTERVENANT - Des photographies ont été prises, en 1942-1943, dans la résidence qui jouxtait Auschwitz où officiers, soldats et personnel divers du camp de concentration venaient se reposer. Ces photographies montrent quelque chose de l'ordre du Club Med, tout le monde vivant une gentille tranquillité. Pensez-vous que la cruauté peut être au fondement des relations humaines? Construire l'humanité, n'est-ce pas justement mener ce combat incessant? On a souvent l'idée d'un monstre qui serait comme un personnage d'opéra chinois, alors que les monstres naissent à l'aune de notre cruauté possible.

Sami Tchak - C'est qui l'humanité ? L'humanité, c'est moi, c'est Hitler, c'est tout le monde. L'humanité n'existe nulle part ailleurs en dehors de ce que les humains sont capables de faire et de ce qu'ils ont fait. Il est clair que la cruauté - ou la violence - est toujours au cœur du fonctionnement de l'humanité ; je ne dis pas de sa construction, qui est tel un volcan. À un moment, il y a une irruption. Quand la lave a fini de couler et de brûler des milliers d'insectes ou d'humains, on vient exploiter les sols fertilisés ; on ne souvient même plus des conséquences. L'humanité fonctionne de cette manière-là.

Un jour, chez Odile Cazenave, à Boston, une jeune Camerounaise a été choquée par ma vision du monde. En effet, quand on parle du rêve américain, se souvient-on que l'on a massacré les Amérindiens pour construire les États-Unis? On ne se pose plus la question des conditions dans lesquelles des pays comme les États-Unis, le Québec ou l'Argentine sont nés. On y a pendu des femmes aux arbres, leurs enfants à leurs pieds, on y a découpé des gens pour nourrir des chiens. Aujourd'hui, on parle des États-Unis comme de la première démocratie du monde, mais on oublie que toute l'Amérique, tous 
les pays américains sont nés non seulement de la traite des Noirs, mais surtout des massacres des Amérindiens, et il n'y a pas si longtemps que ça encore. Lorsque je relis La Destruction des Indes de Las Casas, sa description des massacres commis par les Espagnols dans un village cubain est atroce. Les Espagnols y sont reçus par les Caraïbes qui leur offrent tout ce qu'ils pensent avoir de plus beau. Contrairement aux historiens qui peuvent nous raconter des fables, Las Casas, lui, suivait ceux qui tuaient. Après avoir mangé, il raconte que l'un des Espagnols voulut voir si son sabre était bien aiguisé. Pour ce, il coupa la tête d'un enfant. Finalement, les Espagnols ont massacré tous les habitants du village, parce que l'un d'eux a voulu savoir si son sabre était bien tranchant! Aujourd'hui, lorsque l'on se rend à Cuba, qu'est-ce que ça signifie ? Certes, les Cubains n'ont pas la liberté d'expression, mais c'est une belle île, il y a de la musique !... Mais on a déjà oublié comment est né Cuba. C'est ainsi, l'humanité n'a jamais connu d'époque où la violence n'a pas été à l'origine de la construction de ce dont nous sommes, au bout d'un siècle ou de deux, si fiers.

Les Français, eux, vont parler de leur Révolution, mais si les Vendéens avaient la mémoire de la Révolution, ce ne serait pas pour dire que ce fut beau. Pourtant, tout ce qui est né de la Révolution, nous en sommes les héritiers. C'est une chose absolument universelle. Par exemple, nous les Noirs d'Afrique, on nous a colonisés, on a connu l'esclavage, mais on oublie que notre continent a eu de très grands empires. Or, que ce soit celui du Mali, celui de Chaka Zulu, ou n'importe quel autre empire africain, ils sont nés dans la violence, comme partout ailleurs.

INTERVENANT - Je pensais au lion qui dévore l'antilope. Le lion n'est pas plus cruel que nécessaire : il la dévore et laisse sa carcasse. Vous avez employé l'expression « le monstre de la nuit » et vous avez parlé de jouissance. Dans notre cruauté, si l'on reprend l'image des enfants passés au fil du sabre, la cruauté va bien plus loin que celle du lion, car elle se complait dans la jouissance de son exercice. Dès lors, comment fabrique-t-on notre humanité par rapport à ça et quelle est, en défınitive, la place de l'écrivain?

Sami Tchak - Le lion n'est pas cruel, il fait ce qu'il a à faire pour se nourrir et nourrir ses petits. Par contre, je le trouverais cruel si, pour conquérir une femelle, il tuait les lionceaux qui n'était pas de lui - la femelle devant être disponible pour transmettre ses gènes à lui. C'est un acte simple pour lui, et sans jouissance. Pour nous, humains, c'est insoutenable. C'est là que naît la différence entre les animaux et nous. Nous sommes les seuls êtres doués de raison, mais nous avons oublié que la malédiction inutile de l'être humain, c'est sa raison même. Imaginons tous les monstres ou toutes les monstruosités construites par la raison, cela nous différencie de l'animal. Le lion, comme tous les grands prédateurs, quand il tue ses proies, il ne souhaite pas pour autant les exterminer. Il faut bien que les antilopes se reproduisent pour qu'il puisse se nourrir. Il veille donc à un équilibre. Les humains, quant à eux, peuvent imaginer de les exterminer, c'est la raison, tout est construit par la raison, et l'imagination va la soutenir. À partir de ce moment-là, l'idée de jouissance, selon moi, est strictement humaine. Cela ne me choque pas car je raisonne en termes de civilisation et de société. Que je reste en humanité, cela ne change rien au fonctionnement global d'une civilisation. Actuellement, notre civilisation passe par une sorte de réunification de l'humain au profit du capital. Même si je m'extraie de cette logique, cela ne change rien aux logiques globales : l'argent aujourd'hui est au-dessus des vies humaines. Moi qui reste en humanité, je suis aussi bénéficiaire de cette société.

Un jour, au cours d'un débat, un ami écrivain se faisait le défenseur des faibles en dénonçant les grands groupes qui vont en Afrique pour exploiter les Africains. Or, chez 
moi, au Togo, on n'exploite personne; on n'a rien! Comme il m'a dit offrir des fleurs parfois, je lui ai proposé de lui faire visiter, en Colombie, les lieux de production des fleurs, de voir dans quelles conditions les gens travaillent. Si l'on devait intégrer l'idée de révolte contre tout, jamais nous n'achèterions de fleurs. Je lui ai conseillé de regarder le film Maria pleine de grâce : l'histoire d'une femme qui renonce à récolter des fleurs pour devenir une mule et transporter de la drogue. Pour elle, c'est mieux que de travailler dans les champs. Au-delà de cet exemple, si beaucoup de choses sont à la portée du grand nombre en Occident, dans sa partie la plus riche, c'est bien parce que le monde est mis au service même de l'Occident. On fait travailler des gosses en Asie pour que le prix des chaussures soit ici abordable. Si l'on tient compte de ça, on ne vit plus!

Si nous devons détailler le monde dans sa cruauté, il n'y a peut-être qu'une chose à faire, c'est le quitter. Pour que la révolte soit globale dans la civilisation qui est la nôtre, il faut se suicider, ce que je n'ai pas choisi de faire!

Quel que soit l'humain, même si je sais comment il blesse mon humanité individuelle, je cherche en lui quelque chose qui me touche. Je repense à la polémique autour du film La Chute, un film sur Hitler. Albert Memmi avait été choqué ; il n'avait pas supporté ce moment d'humanité lorsque la mère convainc ses enfants d'accepter la mort. En ce qui me concerne, je voyais juste une mère, prise dans l'étau de l'histoire, qui devait tuer ses enfants. Même s'il s'agissait des enfants de Goebbels, je peux être humainement ému.

Peut-être est-ce ici que l'écrivain a un rôle : non de dire, Hitler ce n'est pas moi, c'est un monstre, mais de montrer en quoi en Hitler il y a une part d'humanité que je porte en moi. Je ne porte pas ses ténèbres, celles qui l'ont conduit à ce que nous savons. Pourtant, même s'il a été le concepteur de ténèbres, je pourrais, à un certain moment, me retrouver en lui. C'est ici tout le processus de la création. Il ne faut pas tomber dans le manichéisme le plus simpliste.

Les véritables créateurs sont ceux qui, en décrivant un saint, peuvent montrer que ce même saint porte aussi en lui la possibilité du pire. Ou que, dans un monstre, il y a toujours une possibilité de la lumière. Il ne faut pas tomber dans le manichéisme le plus simpliste. Je préfère ces œuvres où le contraste en chaque individu, en chaque humain existe. C'est pour cela que je place Dostoïevski au sommet de mon propre panthéon : il sait montrer la beauté de la mouche et la cruauté de l'abeille.

C'est ma vision, on peut lutter tout en ayant suffisamment de lucidité pour savoir que cela ne change rien au système. Gandhi, lui-même, l'a su, le jour où il a reçu une balle en plein public : ce n'est pas parce qu'il a passé sa vie à professer la non-violence que la violence s'arrête.

Ma vision n'inspire pas forcément à l'optimisme : on aime l'humain ou on le quitte.

NICOLAS MARTIN-GRANEL - Tu dis "humain», et dans ta citation tu dis "les vivants de tous les vivants ». Dans un roman de William Boyd, Brazzaville Plage, la femme qui étudie les chimpanzés les voit en train de se déchirer pour le plaisir. II semble y avoir une jouissance.

RAHARIMANANA - Ce qui est étonnant, c'est la définition que vous donnez du mot jouissance. On peut jouir aussi de belles choses, pas seulement de la cruauté.

Sami Tchak - On ne dit pas que l'on jouit uniquement de la cruauté !

RAHARIMANANA - Je comprends ton approche quand tu parles de manichéisme. Mais on peut se dire aussi qu'il y a une forte tendance, dans I'histoire de l'humanité, à aller vers la lumière. Incontestablement, la société est basée sur la violence, la cruauté. Pourtant, la langue que l'on parle, ce n'est pas de la violence, c'est du partage. II y a des cultures que l'on 
partage, des gens qui se rencontrent, certaines structures de sociétés qui se disent : nous sommes ensemble pour que le mieux apparaisse.

Sami Tchak - Pour résumer, partons d'une image simple: on est dans la nuit profonde, éclairés par une chandelle. Si l'on observe bien la lumière que diffuse la chandelle, on remarque une ombre. Il n'existe aucune civilisation qui ait tenté quelque chose de lumineux et qui ne porte en soi déjà cette contradiction. Toute lumière a ses effets pervers. L'idée d'une lumière qui porterait une société n'a jamais existé. Même au cœur de l'ombre qu'est le nazisme, il y a tout de même eu des Allemands portant la lumière. Il ne s'agit pas de souhaiter que l'ombre ou la cruauté soit, il s'agit de reconnaître qu'il n'y a jamais eu d'époque où l'ombre ne fût pas là.

C'est tout. La littérature est là pour nous le rappeler. Tous les grands textes se ressemblent depuis des siècles, car l'humain, qui est leur sujet, n'a pas changé. L'humain ne change pas. Dans mes écrits, j'appelle les contextes historiques «les théâtralités ». Seules les théâtralités contextuelles changent. L'humain, lui, n'a pas changé.

INTERVENANT - Sony Labou Tansi distingue la cruauté du monstre. II n'est pas dualiste. Les humains sont cruels et il invite les monstres froids, des rationalités complètement désincarnées qu'il situe au Nord, à assumer la cruauté. Dans sa pièce Qui a mangé Madame d'Avoine Bergotha ?, il y a ce personnage cruel, Walante, qui fait la morale à des diplomates anglais qui, eux-mêmes, parlent des droits de l'homme. C'est une scène incroyable car la morale est du côté du cruel. Le monstre, c'est un jeune diplomate qui est d'une indifférence totale...

Sami Tchak - La démarche poétique d'un créateur est le réel. Le sociologue, lui, ne juge pas si c'est bon ou si c'est mauvais, il analyse froidement les logiques. Ce n'est pas ce que dit l'artiste. Sony a beau avoir écrit ce qu'il a écrit, il a basculé du côté de l'ombre. En France, on n'en parle pas mais aux États-Unis, on ne cache rien de Sony. Il est humain. Ce que l'artiste dit, ce n'est pas cela que j'analyse. Je pars de l'idée que, dans la société dans laquelle je vis, je n'attends pas que les humains soient parfaits pour les aimer. La personne que je peux suivre dans la rue parce qu'elle m'a fasciné pourra peut-être me tuer. C'est d'ailleurs la première scène de mon livre Filles de mexico, où le Noir rencontre un jeune garçon dont il est tellement amoureux qu'il le suit. Par la suite, le narrateur apprend qu'il l'a tué. Cela ne m'empêchera pas de suivre quelqu'un! Si je dois attendre de quelqu'un qu'il soit parfait, je me poserai alors des questions sur moi-même: est-ce que je suis parfait? Nous parlions de Madagascar 1947. Les cruautés, qui, peut-être, ont été à l'origine de la naissance d'identités collectives ou qui les ont reconfigurées nous frappent plus que les lumières, sinon nous n'en parlerions pas.

INTERVEnANT - Mais dans Madagascar 1947, Jean-Luc essaie de montrer quelles ont été les lumières. Je ne pense pas que ce soit la cruauté qui fascine seulement...

Sami Tchak - Mais d'où vient la lumière car il y a des bourreaux et des résistants ? Si on oublie que la lumière engendre les ténèbres et que les ténèbres engendrent la lumière, on ne dit pas tout. Dans Nour, nous avons tout. Si on chante le résistant, c'est parce qu'il a un bourreau. Dans certains contextes d'ailleurs, on oublie parfois de taire les contrastes des résistants. Prenons le cas de l'Algérie. Lorsqu'on analyse ce qui s'est passé dans ce pays, on ne peut pas dire que la lumière était totalement éblouissante. Dans un contexte historique bien précis, les êtres humains sont capables de tout, quels que soient les objectifs qu'ils poursuivent dans leur lutte. Ce 
n'est pas parce qu'on est résistant que l'on est porteur d'une lumière qui nous écarterait de ce que l'humain peut être capable de faire. Des écrivains essaient d'ouvrir cette plaie-là, comme Anouar Benmalek. Ils disent la complexité du fait humain, du fait historique qui fait que, même dans une lutte absolument noble, des gens peuvent aussi devenir les auteurs d'actes odieux à l'intérieur même des groupes qu'ils sont pourtant censés défendre. On ne peut pas le nier. Le choix de l'artiste, c'est de nous éblouir. Je peux être porté, transporté par n'importe quel poète mais je n'oublie pas le réel. On ne peut oublier qu'au moment où l'Allemagne nazie construisait l'enfer qui est devenu l'enfer européen et mondial, ce pays comptait les écrivains les plus éblouissants qu'il n'ait jamais connus: Thomas Mann, Stefan Zweig... Quand je les lis, j'éprouve vraiment une jouissance, même si je n'oublie pas que l'Allemagne, à ce moment-là, était celle des nazis. Parmi ces écrivains qui m'éblouissent, certains ont dû quitter leur pays, d'autres se suicider.

NICOLAS MARTIN-GRANEL - Il y a quelque temps, je ne sais plus à propos de quel pays ou dictature, tu constatais que les bons écrivains sont souvent sur ce tas de merde.

Sami Tchak - Mikhaill Boulgakov n'aurait peut-être pas écrit Le Maître et Marguerite dans un contexte de démocratie tranquille. Au Congo, les dictatures ont donné les Sony Labou Tansi, Henri Lopes, Tati Loutard. Certains d'entre eux travaillaient avec les dictateurs. Les dictatures africaines, pour ceux qui en parlent, peuvent être considérées comme une simple rigolade par rapport à ce que furent les dictatures latino-américaines. Regardez le nombre de grands écrivains latino-américains qui ont reçu le Nobel, six ou sept. Quand Asturias écrivait, le Guatemala vivait une dictature féroce, ou encore Borges en Argentine, Neruda au Chili...

RAHARIMANANA - N'est-ce pas justement la liberté de ces personnes-là de se dire : je vois la capacité d'être cruel, je vois la capacité d'être dans la lumière, et je fais un choix ? Je ne parle pas ici de l'artiste, mais des sociétés, de l'individu. Je peux choisir entre jouir de l'ombre ou jouir de la lumière. Sinon l'ombre serait toujours la norme. Or, les dictateurs sont tombés, Hitler est tombé, et ils ne sont pas tombés tout seuls. II y a eu des gens qui ont voulu que les choses basculent.

Sami Tchak - Les Français qui ont lutté contre Hitler luttaient pour eux-mêmes, et ensuite ils ont massacré les Malgaches. La France a tout de même entamé ses pires guerres coloniales après Hitler. Il faut lire L'Europe barbare. 1945-1950 de Keith Low. Après la fin de la guerre, l'Europe a connu des cruautés incroyables. Nous n'avons pas mis fin à Hitler parce que nous voulions qu'une civilisation de la lumière règne, il ne faut pas relire l'histoire selon sa vision de poète. Comme l'écrit Césaire dans son Discours sur le colonialisme, on a mis fin à Hitler parce qu'il appliquait aux Occidentaux ce que les Occidentaux eux-mêmes faisaient subir aux autres. C'est tout. Lisez aussi $L a$ Peau de Malaparte qui décrit la cruauté des libérateurs américains vis-à-vis des Européens. On y trouve décrites l'image historique du libérateur mais aussi la réalité de celui qui vient libérer les populations. C'est au cours de cette période que les Américains en ont profité pour imposer certaines normes économiques. L'Europe ne pouvait pas négocier d'égale à égal. C'est quand même l'histoire, et je suis bien plus fasciné par ces contrastes-là de l'histoire que par ma vision poétique. Dans mes livres, je n'appelle pas au triomphe de l'ombre, mais en tant qu'être doué de raison, je reste fasciné par ces contrastes. Je ne peux pas penser que la fin d'Hitler fût liée à quelque idéal que ce soit.

INTERVENANT - Sans faire du psychologisme, il y a quelque chose d'intéressant dans le surcroît de cruauté des troupes françaises visant la répression coloniale après la Deuxième 
Guerre mondiale. II s'agit de tout ce qui a été vécu comme déficit de virilité. Des hommes dévirilisés par la défaite, dévirilisés parce que l'on prenait leurs femmes, etc. On retrouve aussi cette notion de la jouissance.

\section{Sami Tchak - C'est vrai...}

INTERVENANT - Je trouve très intéressant que tu fasses sans cesse le lien entre les hommes et femmes que nous sommes tous et les grands mouvements. On peut aller plus loin et se dire que l'on est tous des êtres vivants concernés par la survie, c'est-à-dire l'adaptation constante à l'environnement. Les espaces de tension qui existent nécessairement ouvrent le champ de l'effervescence des cruautés. C'est là que se joue la question du travail de l'écrivain et de la condition humaine, l'un étant le miroir de l'autre dans toute notre histoire.

RAHARIMANANA - Je persiste à croire qu'il y a des rêves, des idéaux qui construisent les civilisations.

INTERVENANTE - Dans Al Capone le Malien, la quête du balafon va dans ce sens-là, je pense.

Sami Tchak - Dans mes livres, je le redis, je n'appelle pas au triomphe de l'ombre !

RAHARIMANANA - Je ne suis pas en train de te faire ce procès-là mais je trouve que les rêves sont mésestimés. II y a le spectaculaire de la cruauté, on est tous là parce que c'est spectaculaire, on trouve normal que la beauté soit là, que le bien soit là. Mais on n'accorde pas autant d'importance aux petits gestes de tous les jours. Or, ils construisent aussi énormément la société. On est très facilement fascinés, je trouve, par l'ombre, par la cruauté, ce qui peut amener des gens à adhérer à des discours comme celui du nazisme, car il y a cette fascination du spectaculaire. Pourtant, je pense que toutes ces petites choses invisibles qui font aussi notre humanité, si elles ne sont peut-être pas un choc amenant tout de suite à créer une civilisation, une culture, elles restent en revanche absolument fondatrices de beaucoup de choses.

Lorsque j'étais petit, je voyais des gens mourir tout autour de moi. Quand j'ai écrit Lucarne, on m'a dit que j'étais fasciné par la violence, alors que celle-ci me révoltait. Comment faire en sorte, dans un livre, que l'être humain ait la capacité de vivre des petites choses? C'est facile de mettre dans un livre des choses très-très violentes mais travailler le rêve, le beau, l'amour, etc., ce n'est pas simple.

Sami Tchak - Une artiste peintre colombienne a lu nos livres. Jean-Luc et moi nous n'avons pas vraiment la même esthétique. Nous pouvons avoir cette même recherche de lumière, on ne l'exprime pas de la même manière, quelles que soient les ténèbres dont on parle. Cette artiste nous a invités à regarder l'une de ses œuvres intitulée Les Anonymes, puis à choisir un tableau et à écrire librement des textes qu'elle souhaitait intégrer dans son exposition. J'ai donc écrit une sorte de nouvelle. Jean Luc, lui, a écrit plusieurs bouts de textes. Nous ne nous étions rien dit, pourtant une même violence s'est dégagée de nos textes. Pour l'exposition, nous les avons mélangés, ainsi le public ne savait qui avait écrit quoi. Quand nous nous sommes rendus à l'exposition à Saint-Denis, beaucoup de personnes ont trouvé ces textes très violents ; certains détournaient même le regard! Même chose dans la ville du Port, à La Réunion. Les gens se demandaient pourquoi l'artiste avait jugé nécessaire d'associer des textes aussi violents à une si belle exposition ! Pour moi, ce que JeanLuc dit, ce n'est pas vraiment une fascination, c'est plutôt en réalité un malentendu que l'on retrouve à chaque époque. Quand on met à nu la violence pour qu'elle révolte, curieusement elle fascine. On devient complice d'une fascination alors que l'exhibition de ce que l'on porte en soi de sombre est pourtant destinée à nous mettre en garde. J'ai conscience que je porte plusieurs monstres en moi, c'est pour cela que je dois d'abord me méfier de moi avant de me méfier des autres. Si je décris ces monstres-là, c'est pour rappeler aux autres qu'ils les portent aussi et qu'ils doivent 
s'en méfier. Nous sommes nous-mêmes la possibilité du pire. Mais les gens ne lisent pas dans cette optique ; ils lisent avec l'idée que ce qui est écrit, peut-être l'auteur le valide-t-il.

En adoptant une optique holiste, peut-être mon choix me protège-t-il contre moi-même mais pas contre la société, car ses logiques sont au-dessus de moi. Si je ne peux changer la société, ce n'est pas pour autant que je vais suivre passivement ses logiques dominantes. Nous vivons dans une société dont le souci ou l'impératif même d'accumulation de profit et de consommation écrase parfois l'être humain dans sa beauté. Peut-être est-ce pour cela que l'on a cette impression que la lumière n'existe pas.

Il n'y a rien de plus égoïste que l'écriture, car on s'isole dans quelque chose qui est beau pour nous mais qui est absolument inutile. Les écrivains ne contribuent pas à changer quoi que ce soit. Nous ne changeons rien, nous nous changeons nous-mêmes. Écrire est un refuge pour être hors du monde ambiant.

NICOLAS MARTIN-GRANEL - Sony Labou Tansi dit écrire parce que le monde est resté barbare et qu'il n'y a pas de progrès. Peut-être écris-tu pour apporter la lumière qu'il n'y a pas dans le monde, non pour le guérir mais pour au moins l'éclairer.

RAHARIMANANA - Je vais répondre à la place de Sami Tchak. Je pense sincèrement que le livre doit dépasser son auteur. Le discours que tient Sami sur son œuvre n'a aucune incidence sur celle-ci. Un texte vit et nos discours d'auteur ou d'artiste sur nos propres livres sont très vite limités. À chaque fois qu'un livre voyage entre les mains d'un lecteur, il est telle une forêt cachée dans ses mains; chaque lecteur regarde dans sa forêt et fait chanter l'oiseau. Celui-ci peut faire pleurer ou rire. Comment donner à un livre tant de liberté, tant de choix, tant de possibilités d'être ? C'est cela qui est intéressant.

Sami Tchak - Coetzee estime avoir compris une chose dès son premier livre : tout discours que l'écrivain élabore sur ses livres trahit lesdits livres. L'essentiel qu'il a à dire se trouve dans les livres mêmes. Pour lui, aujourd'hui, il vaut mieux défendre les chiens que de s'occuper des humains. Et c'est ce qu'il fait via une association qu'il a créée.

[Entretien transcrit par Greta Rodriguez-Antoniotti]

\section{RÉSUMÉS}

Raharimanana: "J'aimerais orienter mes interventions autour de la complexité de porter les images dans un contexte d'écriture malgache :

* une écriture qui prend ses sources dans la tradition antakarana (voir dans mon roman Revenir) ;

* une écriture qui interroge les images en contexte dominé (colonisation, dictature, voir Madagascar, 1947, Portraits d'insurgés avec Pierrot Men, et Les Cauchemars du gecko et Des ruines)

* une écriture personnelle, de l'artiste que je suis, interroger l'image, Les Cauchemars du gecko, Des ruines. Chacune de mes séquences commencerait avec une lecture musicale et projection des images en question. »

Sami Tchak: "Je pourrais intituler ma communication «L'abeille et la mouche ». Ce sera pour revenir à mon idée exprimée dans Filles de Mexico, puis dans Ainsi parlait mon père, et qui traduit 
ma conception du terrain: Tout créateur est à la fois une mouche et une abeille. En tant qu'abeille, il va aux fleurs, et en tant que mouche, il va à la merde. À lui ensuite, à force de travail, avec persévérance, telles les mouches et les abeilles, de se hisser, à partir de ces matériaux, à la hauteur de l'universelle condition des vivants, de tous les vivants. »

INDEX

Mots-clés : Théâtre, Madagascar, Amérique du Sud, ombre, lumière, images, mémoire, monstres, violence 